bekanntlich ein ebenes Abbild der Kugeloberfläche in Mercator's Projection erzeugt, indem $P$ als Pol betrachtet wird. Durch $\rho=\gamma+i \lg \operatorname{tg} \frac{\sigma}{2}$ wird die Kugel in Bezug auf den Pol $\boldsymbol{I}$ auf dieselbe Weise abgebildet; die vermittelnden Functionen

und

$$
\begin{aligned}
& V=G+i \lg \operatorname{tg} \frac{S}{2} \\
& W=\psi+G+i \lg \operatorname{tg} \frac{S}{2}
\end{aligned}
$$

bilden die Kugeloberfläche ebenfalls in Mercator's Projection $a b$, bezogen auf eine Axe, die senkrecht zur Ebene $P I I$ liegt.

$\mathrm{Zu}$ bestimmen ist noch die Form, welche $H$ annimmt in den vermittelnden Gleichungen

$$
W=\psi+H(\boldsymbol{r})=H(\boldsymbol{\rho}) \text {. }
$$

Man findet sie wohl am leichtesten, wenn man in (ro) statt $\sigma$ und $\gamma$ die Grössen $\varrho=\gamma+i \lg \operatorname{tg} \frac{\sigma}{2}$ und $\gamma$ als Variable einführt und nachher $\gamma=0$ setzt. So erhält man

$$
W=\operatorname{arctg} \frac{\mathrm{x}-e^{-2 i p}}{2 e^{-i \rho}} \quad \text { oder } \operatorname{tg} W=\cos \varrho
$$

Berlin 1892 April.
Zur Ausführung der Coordinaten-Transformation hätte man also der Reihe nach die Gleichungen anzuwenden:

$$
\begin{aligned}
r & =g+i \lg \operatorname{tg} \frac{s}{2} \\
V & =\operatorname{arctg}(\cos r) \\
W & =\psi+V \\
\varrho & =\arccos (\operatorname{tg} W) \\
\gamma+i \lg \operatorname{tg} \frac{\sigma}{2} & =\rho
\end{aligned}
$$

Durch Tabulirung der $V$ als Functionen von $s$ und $g$ würde man demnach gleichzeitig auch einen directen Uebergang von $W$ nach $\sigma$ und $\gamma$ ermöglicht haben.

Es erscheint nicht ausgeschlossen, dass auf Grund solcher oder ähnlicher Ueberlegungen eine Art der Tabulirung ausfindig gemacht werden kann, die vor den bisher gebräuchlichen Methoden, die Transformation sphärischer Coordinaten durch Hülfstafeln zu erleichtern, eine viel grössere Allgemeinheit voraus hat, ohne doch eine zu grosse Ausdehnung der Tafel nothwendig zu machen. Vielleicht ist es der Mühe werth - und aus diesem Grunde habe ich geglaubt obige Darlegungen veröffentlichen zu sollen näher zu untersuchen, ob diese Betrachtungen, die vorerst nur von mathematischem Interesse sind, auch zu einem für die Astronomie und die rechnerische Praxis verwerthbaren Ergebniss zu führen vermögen.

\title{
Cordoba Observations of Comet 1892 I (Swift March 6).
}

\section{By Fohn M. Thome.}

The accompanying observations were begun upon the first clear morning after the receipt of the telegram, and were continued upon every possible occasion; but the weather, (which has been exceedingly bad for a long time) prevented a continuous series, and rendered the observing difficult at times. The nebula and nucleus were both unusually large and bright, but the tail, which I could trace to a distance of $15^{\circ}$ was always faint and indistinct to the naked eye. The nebula, approximately circular, was nearly $5^{\prime}$ in diameter at the end of the series. The nucleus was elliptical, about 1 1" by 8". To the naked eye, the brightness fell between $\theta$ Aquilae and $\beta$ Aquarii, or a little brighter than $3^{\mathrm{d}}$ mag.

The observations were made by me upon the mornings of April 1 and 7 ; by assistant Carl W. Ljungstedt. upon all the other occasions. Equatorial of $\mathrm{I} I$ inches clear aperture and filar micrometer, using a power of 60.

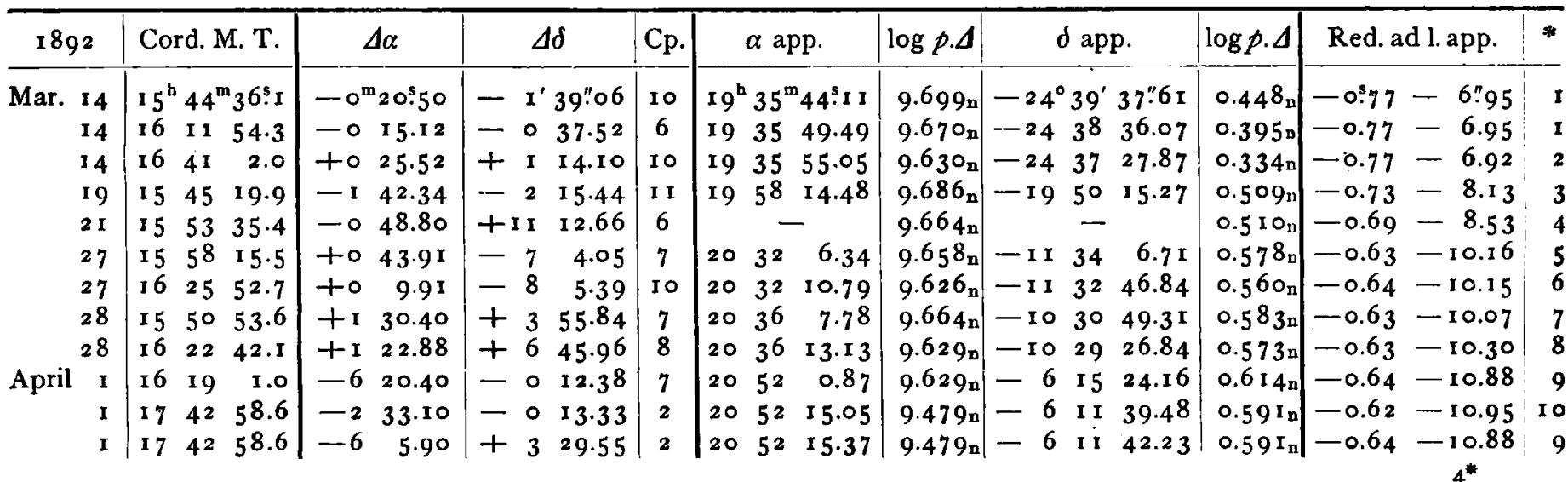




\begin{tabular}{|c|c|c|c|c|c|c|c|c|c|c|c|c|c|}
\hline \multicolumn{2}{|c|}{1892} & Cord. M. T. & $\Delta \alpha$ & \multicolumn{2}{|r|}{$\Delta \delta$} & $\mathrm{Cp}$ & $\alpha$ app. & $\log p \cdot \Delta$ & \multicolumn{2}{|r|}{$\delta$ app. } & $\log p .4$ & Red. ad l. app. & $*$ \\
\hline & 2 & $16^{\mathrm{h}} 12^{\mathrm{m}} 23^{\mathrm{s}} \cdot 7$ & $+0^{m} 59: 57$ & 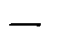 & $3^{\prime} \times 8$ & 10 & $20^{h} 55^{m} 5$ & $9.63^{6}$ & 一 & $5^{\circ} 12^{\prime} 19$ & & -0.59-II"I 4 & 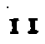 \\
\hline & 2 & $17 \quad 28 \quad 12.7$ & +1 II.1 I & 一 & 0.13 & I 2 & 2056 & $9.5 \circ 3 n$ & - & $8 \quad 58.94$ & $0.607 n$ & $-0.59-\mathrm{I} 1.14$ & I $\mathbf{~}$ \\
\hline & 3 & $\begin{array}{lll}6 & 45 & 20.3\end{array}$ & +130.59 & 一 & 244 & 10 & $20 \quad 5946.02$ & $9 \cdot 59 I_{n}$ & 一 & 743 & $0.626_{n}$ & $-0.58-11.30$ & I 2 \\
\hline & 4 & $\begin{array}{llll}6 & 23 & 1 & 1.0\end{array}$ & to 27.77 & + & 438 & 9 & $\begin{array}{lll}21 & 3 & 29.92\end{array}$ & $9.63^{2} \mathrm{n}$ & 一 & 555 & $0.640_{n}$ & $-0.60-11.37$ & I 3 \\
\hline & 4 & 17 II 7.3 & $-2 \quad 33.03$ & - & 1 I0.10 & 6 & 337.70 & $9.62 \mathrm{r}_{\mathrm{n}}$ & 一 & 356.59 & $0.66 \mathrm{I}_{\mathrm{n}}$ & $-0.5^{8}-11.40$ & 14 \\
\hline & 7 & $17 \quad 1959.0$ & -o 37.62 & + & $1.9^{6}$ & 9 & - & $9 \cdot 524 n$ & & -- & $0.662_{n}$ & $-0.5^{8}-11.88$ & I 5 \\
\hline
\end{tabular}

Mean Places of the Comparison Stars for 1892.0.

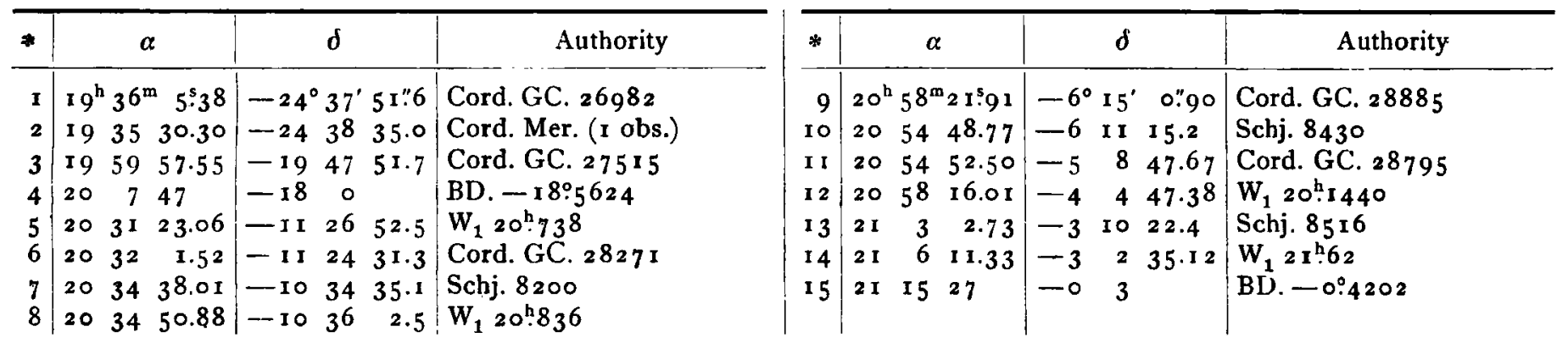

Argentine National Observatory, Cordoba, $189^{2}$ May 1.

Fohn M. Thome.

\section{Observations of Comet $1892 \mathrm{I}$}

made at the Royal Observatory, Cape of Good Hope.

(Communicated by D. Gill Esq. L.L. D., F. R. S., H. M. Astronomer).

\begin{tabular}{|c|c|c|c|c|c|c|c|c|c|c|c|c|c|c|c|c|c|}
\hline \multicolumn{2}{|c|}{1892} & \multicolumn{3}{|c|}{ Cape M. T. } & \multirow{2}{*}{$\frac{\Delta \alpha}{-1^{m} 16^{s} \cdot 4^{2}}$} & \multirow{3}{*}{$\begin{array}{c}\Delta \delta \\
-I^{\prime} 54: 8 \\
-3 \quad 26.6\end{array}$} & \multirow{3}{*}{$\frac{\mathrm{Cp} .}{6.6}$} & \multicolumn{2}{|r|}{$\alpha$ app. } & \multirow{2}{*}{$\frac{\log p \cdot \Delta}{9.602 \mathrm{n}}$} & \multicolumn{3}{|c|}{$\delta$ app. } & \multirow{2}{*}{$\frac{\log p \cdot \Delta}{0.2 \mathrm{I} 3 \mathrm{n}}$} & \multicolumn{2}{|c|}{ Red. ad 1. app. } & \multirow[t]{2}{*}{$*$} \\
\hline & 8 & & & & & & & & & & $-30^{\circ}$ & 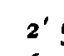 & & & & -5.2 & \\
\hline & 9 & 3 & 31 & $24 \cdot 5$ & & & & & - & - & -29 & I 6 & & $0.453 \mathrm{n}$ & 82 & -5.5 & 2 \\
\hline & 9 & 4 & 41 & 22.0 & 一I 34.78 & & 8 & & I $\mathbf{I}$ & & & - & & & 2 & -5.5 & 2 \\
\hline & Io & 5 & 50 & I $3 \cdot 3$ & + 13.3 & 1-0 20 & 8 & & I 553 & & -28 & 25 & & 0.4 & 3 & $\rightarrow 5$ & 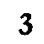 \\
\hline & $\mathbf{I}$ & 4 & 42 & 1.5 & -241 & $-0 \quad 1$ & 8 & & 203 & & -27 & 34 & & $4 \mathrm{n}$ & 3 & -6.0 & \\
\hline & I 6 & 5 & 55 & 44.4 & +249 & 52 & & & 435 & 2 & -22 & 59 & 5 & & 4 & $-7 \cdot 3$ & 5 \\
\hline & 19 & 5 & 54 & 32.7 & & +34 & 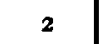 & & & & -20 & 6 & 9.0 & $0.609 \mathrm{n}$ & -0. & -8.1 & \\
\hline & 19 & & 3 & 58.0 & +254 & & 2 & & $56 \quad 5^{6}$ & & & - & & - & -0 & -8.1 & 6 \\
\hline & 20 & 3 & 32 & 48.9 & -0 23 & +234. & .10 & 0 & I 34 & $2 n$ & -19 & 4 & 28.2 & $0.574 \mathrm{n}$ & -0 & -8.4 & 7 \\
\hline & 21 & 5 & $5^{6}$ & 2.1 & - I 47 & -239 & & & - & $9.66 I_{n}$ & & & & & -0.71 & -8.7 & \\
\hline & 23 & & 46 & 25.6 & -I 35.87 & -156.6 & .12 & 20 & 1430 & $9.665 n$ & -16 & 0 & 9.9 & & 69 & -9.1 & 9 \\
\hline & 26 & & 3 & 0.6 & - & -224. & 8 & & - & - & -12 & $5 \mathrm{I}$ & 31.7 & $0.602 \mathrm{n}$ & -0.66 & -9.8 & ro \\
\hline & 26 & I 6 I & I 5 & $5^{8.8}$ & - I 35.78 & & 12 & $2 c$ & 7.04 & $9.629 n$ & & - & & - & -0.66 & -9.8 & 10 \\
\hline & 29 & & 3 & 52.0 & - & $-0 \quad 56.0$ & IO & & - & - & & - & & $0.628_{n}$ & -0.63 & -10.5 & I I \\
\hline & 29 & I 63 & 31 & 48.7 & to 24.18 & - & 6 & & - & $9.604 n$ & & - & & - & -0.63 & -10.5 & 11 \\
\hline & 30 & 16 & 2 & 22.1 & - & - I 22.0 & 4 & & - & & & - & & & -0.63 & -10.6 & 12 \\
\hline & $\mathbf{I}$ & I 6 & I 6 & $55 \cdot 3$ & +123 & -35 & 12 & & $5 \mathrm{I}$ & $9.62 \circ_{n}$ & -6 & 30 & $\cdot 9$ & & $-0.6 I$ & 一II.I & I 3 \\
\hline & 3 & I 6 & 5 & 21.9 & +o 47.77 & -346.9 & 2.12 & 40 & $5^{8} \quad 47.16$ & $9.63 \mathrm{I}_{\mathrm{u}}$ & -4 & 24 & .6 & 0.6 & $-0.6 \mathrm{I}$ & -11.4 & $\mathrm{I} 4$ \\
\hline
\end{tabular}

The observations are corrected for refraction.

Mean Places of the Comparison Stars for 1892.0 .

\begin{tabular}{|c|c|c|c|}
\hline * & $\alpha$ & $\delta$ & Authority \\
\hline I & $19^{\mathrm{h}} \quad 7^{\mathrm{m}} 45^{\mathrm{s}} \cdot 07$ & $-30^{\circ} \quad 0^{\prime} 54^{\prime \prime} 4$ & Stone $1043^{6}$ \\
\hline 2 & 19 I 38.91 & $-2913 \quad 1.2$ & Cord. ZC. $19^{h} \cdot 504$ \\
\hline 3 & 191451.01 & $-28 \quad 25 \quad 39.0$ & $\begin{array}{l}\text { Cord. ZC. } 19^{\mathrm{h}} .599 \text { and cp. } \\
\text { with Cord. ZC. } 19^{\mathrm{h}} \cdot 4^{80}\end{array}$ \\
\hline
\end{tabular}

\begin{tabular}{|c|c|c|c|c|}
\hline * & $\alpha$ & & $\delta$ & Authority \\
\hline 4 & $19^{\mathrm{h}} 23^{\mathrm{m}} 19^{\mathrm{s}} .87$ & $-27^{\circ}$ & $34^{\prime}$ I $0^{\prime \prime} 8$ & Cord. GC. 26690 \\
\hline 5 & $\begin{array}{lll}19 & 41 & 2.34\end{array}$ & -23 & 428.0 & Cord. GC. 27096 \\
\hline 6 & $1954 \quad 3.19$ & -20 & $9 \quad 5.8$ & Cinc. $33^{2} \mathrm{I}$ \\
\hline 7 & $20 \quad 1 \quad 58.59$ & -19 & $\begin{array}{l}6 \\
63.9\end{array}$ & Cinc. 3341 \\
\hline
\end{tabular}

